\title{
Physiological adaptation and transcriptome changes in the plateau plant common vetch (Vicia sativa L.) in response to the plain environment
}

Jinglei He

Northeast Agricultural University

Lingzelai Kong

Northeast Agricultural University

Jinqiu $Y u$

Northeast Agricultural University

Bing Li

Northeast Agricultural University

Xiangyin Hou

Northeast Agricultural University

Yuying Yuan

Northeast Agricultural University

Hang Yin

Northeast Agricultural University

Guowen Cui ( $\sim$ cgw603@163.com )

Northeast Agricultural University https://orcid.org/0000-0003-0496-2840

Research article

Keywords: Qinghai-Tibet Plateau, plain, physiological, transcriptome

Posted Date: February 1st, 2021

DOI: https://doi.org/10.21203/rs.3.rs-191828/v1

License: (1) (1) This work is licensed under a Creative Commons Attribution 4.0 International License. Read Full License 


\section{Abstract \\ Background}

Light and temperature are among the most important factors in plant growth. The annual forage variety common vetch was grown under the special environment of high light intensity and low temperature on the Qinghai-Tibet Plateau. In the seedling stage, the Qinghai-Tibet Plateau adapted variety common vetch was airlifted to the inland plain. Its response to the plain environment and its regulatory mechanism are not clear.

\section{Results}

The samples collected the day before transportation were used as a control. After being transported back to the plain, the morphological structure and physiological indexes were determined on days one, three, five and seven. Compared with the control group, the chlorophyll content in the experimental group changed significantly. Chlorophyll fluorescence increased significantly on the fifth day. The number of stomatal openings decreased significantly on the first day and then increased gradually and exceeded that of the control. Compared with the control sample, the intercellular space of the experimental sample was larger and then did not return to the control level. The physiological indexes gradually returned to the control level on the 7th day. Transcriptome analysis showed that 3251 genes were upregulated and 3317 genes were downregulated on the third day, while 1359 genes were upregulated and 1648 genes were downregulated on the seventh day. These differentially expressed genes were significantly enriched in photosynthesis, photosynthetic antenna protein synthesis, carbon dioxide fixation and chlorophyll synthesis pathways, and almost all of the genes involved in these pathways were downregulated. In addition, MYB, NAC, AP2-EREBP and the Orphans family of transcription factors (TFs) regulating the light response were more abundant on day 3 and day 7 than in the control group.

\section{Conclusion}

The physiological and gene expression levels of common vetch transported to the plain environment were analyzed. To reveal the adaptation mechanism of common vetch to plain environments, the key metabolic pathways of differential gene enrichment were analyzed. These findings are helpful for introducing plateau suitable varieties into plain environments.

\section{Background}

The Qinghai-Tibet Plateau, known as the "third pole of the world" and "Asian water tower", has an average altitude of more than 4000 meters, including vast alpine grasslands [1]. It has the characteristics of low temperature, low oxygen, poor soil and a strong ultraviolet radiation environment. High solar radiation and its effects on secondary metabolites of higher plants in high-altitude areas have been confirmed in 
many studies [2]. In the past 20 years, due to the influence of global climate change, the rate of increase in temperature on the plateau has been much faster than that in most low-lying areas of the world [3-5], and the temperature in the dry period is the limiting factor of vegetation growth in Tibet [6]. The phenology of alpine grasslands has been a hot topic in the scientific community, especially the adaptive genetic mechanism of nonmodel organisms that can be studied in the whole genome by high-throughput sequencing technology [7-8].

Plateau plants have genes that are positively selected, such as those involved in the response to radiation, DNA repair, membrane stability and organic metabolism. These functions may be the reason why plants adapt to high radiation, low temperature and soil depletion environments [9]. When the light needed for photosynthesis is suddenly reduced, the light harvesting complexII (LHCII) can maintain the redox balance between the photosystem I (PSI) and photosystem II (PSII) [10]. The cytochrome (CYP) of different plants may be a positive or negative regulator of drought tolerance. A study on the plateau plant Sophora psammophila showed that drought stress increased the transcription of CYP, and CYP may be an active regulator of drought tolerance [11]. The largest cold-inducible transcription factor (TF) group in Elymus nutans is the AP2/EREBP family. In addition, MADS, Sigma70-like, and Alfin-like also have different expression patterns [12].

Common vetch (Vicia sativa L.) is an annual legume that is widely distributed in the Mediterranean basin, West Asia, Central Asia and China. Because of its strong cold resistance and high seed yield, it is an important economic feed variety for domestic animals to absorb crude protein in high-altitude areas[13]. Research shows that the increase in temperature in the growing season will affect the seed yield and quality of Astragalus sinicus, thus affecting the utilization of Astragalus sinicus [14].

With the increasing demand for animal protein, the demand for animal feed rich in protein is also increasing [15]. However, due to the aggravation of land degradation and the impact of climate change and other factors on the mainland, the original feed crops have been unable to meet the needs of humans. Increasing the basic sources of animal feed and improving utilization efficiency will be of great significance to the development of sustainable animal husbandry [16]. To study the adaptability and regulation mechanism of plateau plants to the plain environment, seedlings of common vetch were transported from the plateau ecosystem to the plain ecosystem. The main objectives are (1) to observe the changes in the morphological structure and leaf structure of plants in the plain environment; (2) to explore the response time of common vetch to adapt to the plain environment through the measurement of physiological indexes; and (3) to identify the different genes involved in the response time to adapt to the plain environment and identify the key genes related to growth and development by combining the results of transcriptome analyses. Then, our goal is to determine the genetic changes in plateau-adapted plants as they grow in plain areas. Then, technical support was provided for introduction in the plain area.

\section{Results}

Changes in chlorophyll content and chlorophyll fluorescence 
Under the plain environment, the chlorophyll content increased significantly on the first and fifth days. Although the control level was restored on day 3, it was lower than that of the control on day 7 (Fig. 1a). Chlorophyll fluorescence (Fv/Fm) was significantly higher on the 5th day and lower on the 7th day than on the day before relocation (Fig. 1b).

\section{Changes in the number of stomata and in the anatomical structure of leaves}

Compared with the control, the number of stomatal openings decreased significantly on the first day, increased significantly on the third day, and reached a relatively stable state on the fifth and seventh days (Fig. 2 a-f). Common vetch is a double-sided leaf with palisade tissue and spongy tissue. On the first day of returning to the plain, the arrangement of upper and lower epidermal cells, palisade cells and spongy cells was loose, and the intercellular space was larger than that of the control. In the next few days, the intercellular space and spongy tissue gradually recovered (Fig. 3 a-e). Therefore, the changes in stomata and anatomical structure showed that the change in common vetch was obvious after it was transported back to the plain, and it was difficult to return to the control state.

\section{Physiological response of common vetch to the plain environment}

Compared with common vetch in the suitable plateau environment (J0), common vetch transported back to the plain showed higher MDA and soluble sugar contents; these contents decreased significantly on the first day (J1) and then increased significantly. The content of soluble protein decreased significantly and then gradually recovered to the same level as on the third day (J3). The levels of $\mathrm{H}_{2} \mathrm{O}_{2}$ and $\mathrm{O}_{2}{ }^{-}$did not change significantly but increased. The activities of POD and GSH decreased significantly on the 5th day (J5) and the 1st day (J1), respectively, but remained the same as the control. The activity of SOD increased significantly; although it decreased significantly on the 5th day (J5), it was still higher than that of the control. In summary, these results show that the damage to the cell membrane and osmotic regulation system of high-altitude adapted species is serious in the plain environment, but it has no significant effect on ROS levels.

\section{RNA sequencing and de novo assembly}

After the seedlings were transported to the plain, the photosynthetic pigment changed significantly on the third day and gradually recovered to stability on the seventh day. Therefore, to obtain the gene expression profile under plain and plateau conditions, the transcriptomes of the leaves of plateau control samples (J0) and plain samples at 3 and 7 days $(\mathrm{J} 3, \mathrm{~J} 7)$ were analyzed via an Illumina platform. From de dovo assembly, a total of 129,209 transcripts and 38,702 unigenes were obtained with an average length of $1552 \mathrm{bp}$. We recorded the N50 value of $2394 \mathrm{bp}$ (Tab. 1). The unigene length ranged from 301 to 16,715 bp. Among the unigenes, 10973 (28.35\%) were more than $2000 \mathrm{bp}$ in length, and there was no single gene shorter than 300 bp. A total of 8789 (22.71\%) were 300-500 bp in length, 9585 (24.77\%) were 500 bp$1000 \mathrm{bp}$ in length, and 9355 (24.17\%) were 1000 bp-2000 bp in length (Tab. 2).

Table 1 Illumina RNA-seq reads and de novo assembly statistics of common vetch. 


\begin{tabular}{|ll|}
\hline Total number of raw reads & $247,896,340$ \\
\hline Total number of clean reads & $60,060,559$ \\
\hline Mean length of reads(bp) & 1919 \\
\hline Number of total unigenes & 38,702 \\
\hline Mean length of unigenes (bp) & 1552 \\
\hline Minimum unigene length (bp) & 301 \\
\hline Maximum unigene length (bp) & 16,715 \\
\hline N50 (bp) & 2394 \\
\hline
\end{tabular}

Table 2 Length distribution of the unigenes.

\begin{tabular}{|lllllll|}
\hline Length $(\mathrm{nt})$ & Total & $<300$ & $300-500$ & $500-1000$ & $1000-2000$ & $\geq 2000$ \\
\hline Number of Unigenes & 38702 & 0 & 8789 & 9585 & 9355 & 10973 \\
\hline$\%$ & $100 \%$ & $0 \%$ & $22.71 \%$ & $24.77 \%$ & $24.17 \%$ & $28.35 \%$ \\
\hline
\end{tabular}

\section{Functional annotation}

Among the 38,702 unigenes, a total of 29,384 unigenes were annotated in at least one database, including 25,225 unigenes annotated in the NR database, 25,201 unigenes in NT, 18,771 unigenes in PFAM, 18,771 unigenes in GO, and 6042 unigenes in the KOG database (Fig. 5a).

Compared with the annotation results of the NR database, it can be seen that the most homologous species with common vetch is Medicago truncatula, with 8391 species (33.3\%). In addition, $18.4 \%$ of unigenes were similar to those of Trifolium pratense (Additional file 1: Figure S1.). In accordance with GO analysis, 105146 single genes were classified according to the three major categories of GO. There were 25 groups of biological processes (BPs), 20 groups of cellular components (CCs) and 10 groups of molecular functions (MFs) (Fig. 5b, additional file 2: Table S1). The most abundant GO terms in the unigenes of common vetch were "metabolic process" (10131), "cellular process" (11068) and "singleorganism process" (8056) for BP; "cell part" (6127) and "cell" (6127) for CC; and "catalytic activity" (8350) and "binding" $(10,690)$ for MF.

After KEGG annotation, 8185 DEGs were mapped into 130 KEGG metabolic pathways. The top three KEGG pathways were "metabolism" (4870), "genetic information processing" (2099) and "environmental information processing" (412). The proportion of genes in the total number of annotated genes was ribosome (ko03010, 510 single genes), plant pathogen interaction (ko04626, 304 single genes), carbon metabolism (ko01200, 3011 single genes) and plant hormone signal transduction (ko04075, 274 single genes) (Fig. 5c, Additional file 3: Table S2.). 


\section{Overall analysis of DEGs}

In this experiment, we used deseq software to analyze the differential expression among the sample groups, with FDR $<0.05$. Compared with the control (J0), samples collected on the third day (J3) had 3251 upregulated genes and 3317 downregulated genes, and 1359 upregulated genes and 1648 downregulated genes were observed on the seventh day (Fig. 6a). A total of 443 genes showed the same change in three comparisons when comparing the DEGs on different days. Compared with the control (J0), there were 1491 DEGs on the 3rd and 7th days (Fig. 6b). To confirm this result, 8 genes with different transcription abundances were verified by real-time RT-PCR. The gene expression levels obtained with the two methods were consistent.

In the J3 vs J0 comparison, the top seven positions of the KEGG enrichment pathway are shown in Fig. 7a, Additional file 4: Table S3 (Q value < 0.01), namely, 28 in photosynthesis (ko00195), 15 in photosynthetic antenna protein (ko00196), 25 in photosynthetic biological carbon fixation (ko00710), 16 in phenylalanine metabolism (ko00360), 15 in porphyrin and chlorophyll metabolism (ko00860), and 14 in tyrosine metabolism (ko00350).

In the J3 vs J0 comparison, the top four positions of the KEGG enrichment pathway are shown in Fig. 7a, Additional file 5: Table S4 (Q value < 0.01), 206 in ribosomes (ko03010), 36 in photosynthesis (ko00195), 43 in ribosome biogenesis in eukaryotes (ko03008), and 21 in photosynthesis-antenna proteins (ko00196).

The pathways photosynthesis (ko00195) and photosynthesis-antenna proteins (ko00196) were identified in the $\mathrm{J} 3$ vs $\mathrm{J} 0$ and $\mathrm{J} 7 \mathrm{vs} \mathrm{J} 0$ comparisons. The pathways identified in only the $\mathrm{J} 3$ vs $\mathrm{J} 0$ comparison were carbon fixation in photosynthetic organisms (ko00710), phenylalanine metabolism (ko00360), porphyrin and chlorophyll metabolism (ko00860), and tyrosine metabolism (ko00350). Pathways specifically in J7 vs $\mathrm{J0}$ are ribosome (ko03010) and ribosome biogenesis in eukaryotes (ko03008).

\section{Differential expression of photosynthesis-related genes}

To study the adaptability of plateau plants to the plain environment, genes related to environmental effects were screened out from the filtered DEGs for further study. In J3 and J7, DEGs related to the reaction center of the PSI and PSII, photosynthetic antenna protein, electron transfer and $\mathrm{CO}_{2}$ fixation were identified. The main pathways were photosynthesis (k00195), photosynthesis antenna protein (ko00196) and photosynthesis biological carbon fixation (ko00710). When the plants were transported to the plain environment, the carbon fixation of photosynthetic organisms was inhibited. The main feature is that the core cytochrome $b_{6} / f(k 02636)$, which connects PSII and PSI, is downregulated during electron transfer. During photophosphorylation, the genes encoding FD-NADP ${ }^{+}$reductase $(k 02641)$ and ATP synthase (k02113, k02109) were downregulated (Fig. 8b).

During the Calvin cycle (Fig. 8a), the genes encoding Rubisco (k01601) in the pathway of 3phosphoglycerate (PGA) synthesis were downregulated. The genes encoding phosphoglycerate kinase 
(k00927) and glyceraldehyde-3-phosphate dehydrogenase (k05298) were downregulated in the synthesis of 3-phosphoglyceraldehyde (GAP). Many enzymes involved in the regeneration of ribulose-1,5disphosphate (RuBP) were downregulated. During the $\mathrm{C} 4$ cycle, oxaloacetic acid (OAA) is reduced to malic acid; the gene encoding malate dehydrogenase (K00025) is downregulated. Regarding regeneration, the genes encoding pyruvate transaminase (k00814) were downregulated. However, the genes encoding aspartate transaminase ( $\mathrm{k14454)}$, involved in transamination, were upregulated in the J3 vs J0 comparison (Fig. 8d). Lhca2, Lhca4, Lhcb1, Lhcb2, Lhcb3, Lhcb4, Lhcb5 and Lhcb6 in the photosynthetic antenna protein pathway, components of the complementary light complexes LCHI and LCHII, were downregulated. In addition, the genes encoding proteins in the reaction center of photosynthesis were downregulated, including PSI reaction center subunits III-VI (PsaF, PsaE, PsaG, PsaH), PSI reaction center subunits $\mathrm{X}$ and $\mathrm{XI}$ (PsaK, PsaL), PSI reaction center subunits PsaN and PsaO, and PSIl reaction center PsbK, PsbO, PsbQ, PsbW, and Psb27. In conclusion, the downregulation of these three pathways inhibited the growth of common vetch in the plain environment.

\section{Differential expression of encoding photosynthetic pigment-related genes}

The adaptability of plants to light is closely related to the photosynthetic pigment production pathway (Fig. 8c). In this study, the DEGs were mainly in the porphyrin and chlorophyll metabolism (ko0860)

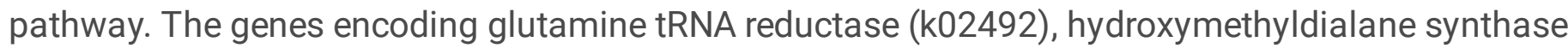
(k01749), uroporphyrinogen decarboxylase (k01599), Mg-chelatase subunit H (k03403), magnesiumprotoporphyrin 0-xymethyltransferase (k03428), magnesium-protoporphyrin IX monomethyl ester (oxidative) cyclase (k04035), protochlorophyllide reductase (k00218), chlorophyll An oxygenase (k13600) and chlorophyll a synthase ( $\mathrm{k} 04040$ ) were downregulated in J3 compared with the control.

\section{RNA-seq validation: qRT-PCR analysis}

We used qRT-PCR to detect the consistency of the expression patterns of 8 randomly selected genes. GAPA (Cluster-7705.13598, Cluster-7705.13381, Cluster-7705.13418), rbcS (Cluster-7705.13113), ATPF0B (Cluster-7705.13726), ATPF1D Cluster-7705.13438), petH (Cluster-7705.13612), and petC (Cluster7705.14151) were found to be downregulated in J3 vs J0 and J7 vs J0 by qRT-PCR. The qRT-PCR results for all genes were consistent with those of RNA-Seq, which provided strong credibility for our RNA-Seq data. (Fig 9)

\section{DEGs encoding TFs}

There were more TFs in $\mathrm{J} 7$ than in J3. In J3, 109 upregulated TF genes were divided into 39 families, mainly NAC (11.01\%), Orphans (7.34\%), and HB (7.34\%), and 94 downregulated genes were divided into 32 families, including MYB (15.96\%), AP2-EREBP (10.64\%), and orphans (8.51\%) (Fig. 10a, Additional file 6: Table S5.). However, 206 upregulated TF genes in $\mathrm{J7}$ were divided into 52 families, mainly AP2-EREBP (12.62\%), mTERF (8.25\%), MYB (7.77\%), and Orphans (5.34\%), and 238 downregulated genes were divided into 40 families, including MYB (13.45\%), AP2-EREBP (9.66\%), and NAC (8.40\%) (Fig. 10b, Additional file 7: Table S6.). These results indicate that among all of these differentially expressed TFs, a 
large number of TFs of the MYB, NAC, AP2-EREBP and Orphans families were differentially expressed regardless of the number of days of adaptation after the move to the plain environment. These TFs may play an important role in the acclimation of common vetch to the plain environment.

\section{Discussion}

\section{Chlorophyll synthesis, stomata and leaf structure of common vetch in the plain environment}

Light is the direct energy source of plant growth. Among them, light intensity is one of the most important factors that affect many physiological processes of plants, such as photosynthesis $[17,18]$. There is a threshold for the effect of light intensity on the growth of plants; light intensity that is too high or too low will inhibit the growth of plants [19]. The results showed that chlorophyll content was positively correlated with $\mathrm{CO}_{2}$ concentration and light intensity [20]. Higher chlorophyll content, higher stomatal density and thicker leaves were beneficial for increasing the photosynthetic rate. Under shading conditions, the stomatal density of soybean decreased significantly [21,22]. In this study, the chlorophyll content in the leaves of common vetch decreased significantly in the plain environment compared with the plateau environment, which indicated that photosynthesis was inhibited under low light conditions in the plain. At first, when stimulated by the environment (J1), the stomata closed, and the intercellular space became larger, representing the response of common vetch to a stressful environment. To adapt to the plain environment, the above indexes gradually recovered to stable levels. In addition, there is a close relationship between the activity of the chlorophyll cycle and the construction and destruction of photosynthetic organs. The lack of chlorophyll b resulted in a significant decrease in lhcb1, Lhcb6 and Ihca1 in LHC subunits $[23,24]$. ChlH is a porphyrin-binding subunit and a key enzyme in chlorophyll synthesis [25]. Mg-chelatase makes protoporphyrin IX participate in chlorophyll biosynthesis, and its activity decreases with decreasing $\mathrm{ChlH}$ transcription levels [26]. In this study, $\mathrm{ChlH}$ and other genes involved in chlorophyll synthesis (HemA, HemC, HemE) were downregulated. The above results were consistent with the chlorophyll content results (Fig. 11c).

\section{Adaptive regulation of photosynthesis in the plain environment}

Photosynthesis usually refers to the process by which green plants absorb light energy, synthesize carbon dioxide and water into energetic organic matter, and release oxygen at the same time. It is also an important factor that affects plant growth, yield and fruit quality. The differences in genes among different varieties result in differences in photosynthesis. Therefore, more attention should be paid to improving the photosynthesis of plants in actual agricultural production $[27,28]$. The electron transport system of water molecule oxidation and release is transferred to NADP+ and reduced to NADPH, and the cycle of electron transport is driven by light reactions. The electrons produced by water splitting in PSII are transferred to ferritin ( $F d$ ) through cytochrome (Cyt) $b_{6} / f$ and PSI; thus, NADPH is produced. Cyt- $b_{6} / f$ plays a unique role in photosynthetic electron transport. It can act on both linear electron transport (production of ATP and NADPH) and cyclic electron transport (production of ATP only) [29]. The cytochrome $b_{6} / f$ complex and chloroplast ATPase are the main sites that control photosynthetic flux. The 
ability of protons to enter the lumen is controlled by the electron flow of the Cyt- $\mathrm{b}_{6} / \mathrm{f}$ complex, and the proton flow through ATPase is balanced. ATP synthases drive ATP synthesis [30]. It has been reported that the content of the $\mathrm{Cyt}^{-\mathrm{b}_{6}} / \mathrm{f}$ complex in Arabidopsis thaliana is positively correlated with an increase in the light intensity for constant growth, that is, high light > normal light > low light [31]. It can be inferred

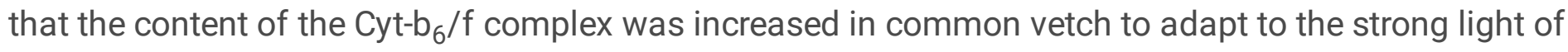
the Qinghai-Tibet Plateau. After moving to the plain, the light intensity decreased, and the content of the $\mathrm{Cyt}-\mathrm{b}_{6} / \mathrm{f}$ complex decreased. This is consistent with the transcriptome sequencing results. The decrease in the Cyt- $b_{6} / f$ complex reduces the electron transport rate of PSII, which leads to a decrease in the $\mathrm{CO}_{2}$ assimilation rate [32]. The results show that $C y t-b_{6} / f$ has a high level of control over the electronic transmission rate of Setaria viridis in C4 plants [33], and high Cyt- $\mathrm{b}_{6} / \mathrm{f}$ abundance in $\mathrm{C} 4$ plants may lead to a high $\mathrm{CO}_{2}$ assimilation rate and high yield [34]. In plants, NADP+ reductase (FNR) receives electrons from FD at the end of the electron transport chain of photosynthesis and converts NADP+ to NADPH for $\mathrm{CO}_{2}$ fixation and chloroplast metabolism [35]. In pea leaf experiments, it was suggested that the activation of FNR and the increase in electron flow between PSII and PSI could lead to the acceleration of NADP+ reduction by FD [36]. ATPase plays a key role in the coregulation of light and dark reactions in photosynthesis. Experiments have suggested that photosynthesis can be controlled by regulating ATPase in tobacco under the conditions of high $\mathrm{CO}_{2}$ and low $\mathrm{O}_{2}$ [37] (Fig. 11a-b).

Ribulose 1,5-diphosphate carboxylase/oxygenase (Rubisco; EC 4.1.1.39) fixes carbon dioxide in the atmosphere to participate in the Calvin cycle of photosynthesis. Rubisco immobilizes carbon dioxide to form two 3-phosphoglycerate (PGA) molecules. The formed 3-PGA forms glyceraldehyde-3-phosphate through two reactions of ATP consumption and NADPH consumption. The regeneration stage of the cycle includes a series of reactions to form phosphorylation. Finally, ribose-5-phosphate kinase (Ru5PK) catalyzes the formation of RuBP by consuming 1 molecule ATP. The whole process requires the consumption of two molecules of NADPH and three molecules of ATP [38,39]. common vetch belongs to the C4 plant of the NAD-ME type, which uses aspartic acid as the main transport metabolite. Aspartic acid is converted to malic acid by reductive deamination [40]. It has been confirmed that the Rubisco content of sunflower (Helianthus annuus L.) decreased under shading [41] (Fig. 11d).

The Rubisco content of rice decreased significantly under high temperature [42]. However, in this experiment, compared with the control, 19 genes in J3 and J7 were involved in carbon fixation in photosynthetic organisms, and all of them were downregulated, including six downregulated genes encoding rbcL and rbcS. This is suitable for highland common vetch in environments of low light intensity and low $\mathrm{CO}_{2}$ concentrations in the plain. These genes limit the photosynthesis and carbon-fixing capacity of common vetch by reducing the efficiency of electron transport.

\section{Regulation of antioxidant capacity in the plain environment}

TFs are proteins that can regulate gene expression [43]. TF gene expression regulation can make plants respond to changes in the external environment in a highly specific and flexible way [44]. Furthermore, the 
environment can promote the activity of photosynthetic genes and improve the photosynthetic efficiency of plants [45]. To better understand the regulation process of common vetch under low light and low $\mathrm{CO}_{2}$ concentrations, we identified a large number of candidate TFs that directly or indirectly participate in the transcription of genes related to the light response. It is noteworthy that the TF family related to MYB, NAC, AP2-EREBP and Orphans was differentially expressed.

\section{Conclusions}

The results showed that the plateau plant common vetch could adapt by changing its morphological and physiological characteristics when it was transported to the plain environment by air at the seedling stage. A large number of DEGs were identified by RNA-Seq in pea. These DEGs were mainly downregulated and involved in photosynthesis and carbon dioxide fixation pathways. TFs regulate the expression of light-responsive genes to allow plants to enter the plain environment.

\section{Methods}

\section{Plant materials}

The experiment was carried out in the field of Sangzhuzi District, Tibet Autonomous Region in 2019. The seeds of common vetch ( $V$. sativa), that is, XiMu432, by Qinghai Academy of Animal Husbandry and Veterinary Sciences, were seeded in a round plastic basin (diameter: $10 \mathrm{~cm}$ and height: $15 \mathrm{~cm}$ ). The soil was a uniform nutrient soil. The plants were transported in the basin during the seedling stage to the outdoor experimental site of Northeast Agricultural University of Harbin City by air (Additional file 8: Table S7). Samples were collected on the first, third, fifth and seventh days (J1, J3, J5, and J7, respectively). The samples collected before high-altitude transportation were set as the control (J0). After sampling from 9:00 to 11:00, the plants were frozen in liquid nitrogen and stored in a refrigerator at $-80^{\circ} \mathrm{C}$, and three independent samples were collected daily for physiological index measurements. The samples collected on the $3 \mathrm{rd}$ and 7 th days were used for transcriptome sequencing.

\section{Measurement of stomatal density and leaf structure}

Fresh leaves were cut into $2 \times 5 \mathrm{~mm}$ strips and stored in glutaraldehyde. The leaves were dehydrated with a series of solutions. The stomatal opening and closing of leaves were observed with an S-3400N (Hitachi, China) scanning electron microscope. Five field images were saved for each sample, and the number of stomata was calculated.

The veinless leaves of common vetch were fixed in FAA solution to prepare paraffin patches $(3 \times 5 \mathrm{~mm})$ and stained with solid green and safranin. The cross sections of $10 \mu \mathrm{m}$ thick leaves were analyzed under a 10 -fold microscope (Nikon Eclipse 50i, Japan) to observe the state of palisade tissue and spongy tissue. 
Chlorophyll fluorescence (Fv/Fm) was measured by a portable photosynthetic apparatus after 30 minutes of leaf shading. After the samples were transported back to the laboratory, physiological indexes, namely, the osmotic regulation system (soluble sugar, soluble protein), antioxidant system (SOD, POD, CAT), oxidation system $\left(\mathrm{O}_{2}, \mathrm{H}_{2} \mathrm{O}_{2}\right)$ and MDA, were determined with a test kit (Comin, Suzhou, China).

\section{RNA extraction and library preparation for transcriptome sequencing}

Total RNA was isolated from common vetch by using the NEBNext@ Ultra ${ }^{\text {TM }}$ RNA Library Prep Kit (NEB, USA). Agarose gel electrophoresis ( $1 \%$ gel concentration) and a NanoPhotometer ${ }^{\circledR}$ spectrophotometer (IMPLEN, CA, USA) were used to detect whether RNA was degraded and RNA purity. The cDNA library was prepared with the NEBNext ${ }^{\circledR}$ Ultra ${ }^{\text {TM }}$ RNA Library Prep Kit for the Illumina sequencing system (NEB, USA). The mRNA was isolated and purified from total RNA by magnetic beads linked with poly-T oligosaccharides. The first cDNA was synthesized from the interrupted mRNA, and then the second strand cDNA was synthesized by DNA Polymerase I and RNase H. An Ampure XP system (Beckman Coulter, Beverly, USA) was used to select the fragment size (250-300 bp), and PCR was used to enrich the cDNA library. The quality of the constructed library was evaluated by an Agilent Bioanalyzer 2100 system. Finally, the Illumina HiSeq platform was used for high-throughput sequencing. Transcriptome datasets can be used in the NCBI Sequences Read Archive under accession number PRJNA688111.

\section{Functional annotation and differentially expressed gene (DEG) analysis}

Use the FASTX toolkit to read the clean sequence. The Trinity program was used for the assembly of single genes. The NR, NT (http://www.ncbi.nlm.nih.gov/), PFAM (http://pfam.sanger.ac.uk/), GO (http://www.geneontology.org/), and KOG (http://www.ncbi.nlm.nih.gov/COG/) databases were used to annotate gene functions to obtain gene annotation functions. Differential expression analysis of two samples was performed using the DEGseq (2010) R package. P value was adjusted using q value. $q$ value $<0.005 \& \mid \log 2 \mathbb{X f o l d}$ change $\mathbb{|} \mid>1$ was set as the threshold for significantly differential expression. All the DEGs were subjected to enrichment analysis according to GO functions and KEGG pathways.

\section{Quantitative real-time PCR (qRT-PCR) analysis}

To confirm the DEG results, 8 transcripts were randomly selected from the photosynthesis pathways (ko00195) and carbon fixation in photosynthetic organisms pathways (ko00710) for qRT-PCR verification. Primer 5 software was used to design gene-specific qRT-PCR primers (Attached file 9: table S8).

The LightCycler Multiplex DNA Master Kit (CWBIO, China) used for qRT-PCR quantitative analysis was completed. The $10 \mu \mathrm{L}$ reaction mixture was $5 \mu \mathrm{L}$ of $5 \mathrm{X}$ reaction mixture, $3.6 \mu \mathrm{L}$ of distilled water, $1 \mu \mathrm{L}$ of cDNA and $0.4 \mu \mathrm{L}$ of each primer $(10 \mathrm{mM})$. qRT-PCR analysis was performed on a LightCycler 480 realtime PCR system (Roche, Switzerland) $\left(95^{\circ} \mathrm{C}\right.$ for 3 minutes, 40 cycles of $95^{\circ} \mathrm{C}$ for 10 seconds, $58^{\circ} \mathrm{C}$ for 30 seconds, $72^{\circ} \mathrm{C}$ for 30 seconds). The $2^{-\Delta \Lambda} \mathrm{CT}$ calculation method was used to analyze the gene expression data. 


\section{Statistical analysis}

SPSS 19.0 software was used to perform one-way analysis of variance (ANOVA) to evaluate the difference between the plateau condition and the control treatment. ANOVA with Duncan's comparison was considered to show significant differences at the $5 \%$ level in all comparisons. At least three biological replicates were used for each statistical analysis in controls and samples. Microsoft Excel 2016 was used for data analysis, and SigmaPlot was used to draw the graphs.

\section{Abbreviations}

MDA: malondialdehyde; POD: Peroxidase; SOD: Superoxide dismutase; $\mathrm{GSH}$ : glutathione; $\mathrm{H}_{2} \mathrm{O}_{2}$ : hydrogen peroxide; $\mathrm{O}_{2}^{-}$: superoxide anion; BP: Biological process; $\mathrm{CC}$ : Cellular component; MF: molecular function; DEG: Differentially expressed genes; TF: transcription factors; NR: NCBI nonredundant protein; NT:

Nucleotide Sequence Database; GO: Gene Ontology; KOG: EuKaryotic Orthologous Groups; PFAM: Protein family; KEGG: Kyoto Encyclopedia of Genes and Genomics; qRT-PCR: Quantitative real-time RT-PCR.

\section{Declarations}

\section{Ethics approval and consent to participate}

Not applicable.

\section{Consent to publication}

Not applicable.

\section{Competing interests}

The authors declare that they have no competing interests.

\section{Availability of data and materials}

Raw reads of one Illumina RNA-seq library generated in this study are available from BioProject at NCBI (https://www.ncbi. nlm.nih.gov/bioproject/) under accession number PRJNA688111.

\section{Funding}

This research was funded by the National Natural Science Foundation of China (31872998). The funding organizations provided financial support to the research projects but were not involved in the design of the study, data collection, analysis of the data, or writing of the manuscript.

\section{Authors' contributions}


GW designed the research. JL carried out the experiments with the help of ZL, JQ, BL, XY, YY and HY. JL collected the experimental data and drafted the manuscript. GW reviewed the manuscript and part of the data analysis. All authors read and approved the final manuscript.

\section{Acknowledgments}

Not Applicable.

\section{References}

1. Li G, Jiang C, Cheng T, et al. Grazing alters the phenology of alpine steppe by changing the surface physical environment on the northeast Qinghai-Tibet Plateau, China. Journal of Environmental Management. 2019, 248(Oct.15):109257.1-109257.7.

2. Senica M, Stampar F, Veberic R, et al. The higher the better Differences in phenolics and cyanogenic glycosides in Sambucus nigra leaves, flowers and berries from different altitudes. Journal of the ence of Food \& Agriculture. 2017;97(8):2623-2632.

3. Pepin N, Bradley R S, Diaz H F, et al. Elevation-dependent warming in mountain regions of the world. Nature Climate Change. 2015;424-430.

4. Piao $S$, Ciais $P$, Huang $Y$, et al. The impacts of climate change on water resources and agriculture in China. Nature. 2010;467(7311):43-51.

5. Ke H, Yangjian Z, Juntao Z, et al. The Influences of Climate Change and Human Activities on Vegetation Dynamics in the Qinghai-Tibet Plateau. Remote Sensing. 2016;8(10):876.

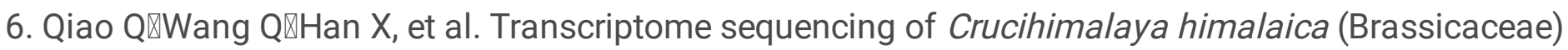
reveals how Arabidopsis close relative adapt to the Qinghai-Tibet Plateau. Nature.2016.

7. Shipeng Yang, Xuemei Sun, Xiaoting Jiang, et al. Characterization of the Tibet plateau Jerusalem artichoke (Helianthus tuberosus L.) transcriptome by de novo assembly to discover genes associated with fructan synthesis and SSR analysis. 2019;156(1).

8. Zhou D, Gao S, Wang H, et al. De novo sequencing transcriptome of endemic Gentiana straminea (Gentianaceae) to identify genes involved in the biosynthesis of active ingredients. Gene. 2016;575(1).

9. Qiao Q, Wang Q, Han X, et al. Transcriptome sequencing of Crucihimalaya himalaica (Brassicaceae) reveals how Arabidopsis close relative adapt to the Qinghai-Tibet Plateau. Rep. 2016;6:21729.

10. M. Pietrzykowska, M. Suorsa, D. A. Semchonok, et al. The Light-Harvesting Chlorophyll a/b Binding Proteins Lhcb1 and Lhcb2 Play Complementary Roles during State Transitions in Arabidopsis. The Plant Cell, 2014;26: 3646-3660.

11. Huie L, Weijie Y, Yaru F, et al. De Novo Assembly and Discovery of Genes That Are Involved in Drought Tolerance in Tibetan Sophora moorcroftiana. Plos One. 2015; 10(1): e111054.

12. Fu J, Miao Y, Shao L, et al. De novo transcriptome sequencing and gene expression profiling of Elymus nutans under cold stress. Bmc Genomics. 2016, 17(1):870. 
13. Tae-Sung K, Sebastin R, Sundan S, et al. Transcriptome Analysis of Two Vicia sativa Subspecies: Mining Molecular Markers to Enhance Genomic Resources for Vetch Improvement. Genes. 2015; 6(4):1164-1182.

14. Li R, Chen L, Wu Y, et al. Effects of Cultivar and Maternal Environment on Seed Quality in Vicia sativa. Frontiers in Plant ence. 2017; 8:1411-1419.

15. Aouida M, Rook F, Bianca Maimann A, et al. Polymorphisms in cyanogenic glucoside and cyanoamino acid content in natural accessions of common vetch (Vicia sativa L.) and selection for improved agronomic performance. Plant Breeding. 2019.

16. Makkar H P S, Tran G, Gigerreverdin S, et al. Seaweeds for livestock diets: A review. Animal Feed ence \& Technology. 2016; 212:1-17.

17. Lin K H, Shih F C, Huang M Y, et al. Physiological Characteristics of Photosynthesis in Yellow-Green, Green and Dark-Green Chinese Kale (Brassica oleracea L. var. alboglabra Musil.) under Varying Light Intensities. Plants. 2020.

18. Wang C L, Guo Q S, Zhu Z B, et al. Physiological characteristics, dry matter, and active component accumulation patterns of Changium smyrnioides in response to a light intensity gradient[J]. Pharmaceutical Biology. 2017;55(1):581.

19. Meng, ZJ, Zhang, et al. Subhigh Temperature and High Light Intensity Induced Irreversible Inhibition on Photosynthesis System of Tomato Plant (Solanum lycopersicum L.). FRONT PLANT. 2017;8:365381.

20. Huang W, Han S, Zhou Q, et al. Assessing interactions between environmental factors and aquatic toxicity: Influences of dissolved $\mathrm{CO}_{2}$ and light on $\mathrm{Cd}$ toxicity in the aquatic macrophyte Potamogeton crispus. Aquatic Toxicology. 2019;212.

21. Fan Y, Chen J, Wang Z, et al. Soybean (Glycine max L. Merr.) seedlings response to shading: leaf structure, photosynthesis and proteomic analysis. BMC Plant Biology. 2019; 19(1).

22. Jumrani K, Bhatia V S. Influence of different light intensities on specific leaf weight, stomatal density photosynthesis and seed yield in soybean. 2020.

23. Sato R, Ito H, Tanaka A. Chlorophyll b degradation by chlorophyll b reductase under high-light conditions. Photosynthesis Research. 2015;126(2):249-259.

24. Tyutereva E V, Evkaikina An I, Ivanova A N, et al. The absence of chlorophyll b affects lateral mobility of photosynthetic complexes and lipids in grana membranes of Arabidopsis and barley chlorina mutants. Photosynthesis Research. 2017.

25. Xiaoqin L, Yue L, Shangwei Z. Interplay between Light and Plant Hormones in the Control of Arabidopsis Seedling Chlorophyll Biosynthesis. Frontiers in Plant ence. 2017;8:1433.

26. Luo S, Luo T, Liu Y, et al. N-terminus plus linker domain of Mg-chelatase D subunit is essential for Mg-chelatase activity in Oryza sativa [J]. Biochemical \& Biophysical Research Communications. 2018;497(2):749. 
27. Huang W, Han S, Zhou Q, et al. Assessing interactions between environmental factors and aquatic toxicity: Influences of dissolved $\mathrm{CO}_{2}$ and light on $\mathrm{Cd}$ toxicity in the aquatic macrophyte Potamogeton crispus. Aquatic Toxicology.2019;212.

28. Gitelson A A, Peng Y, Arkebauer T J, et al. Productivity, absorbed photosynthetically active radiation, and light use efficiency in crops: Implications for remote sensing of crop primary production. Journal of Plant Physiology. 2015;177:100-109.

29. Suzuki, Yuji, Kondo, et al. Enhanced leaf photosynthesis as a target to increase grain yield: insights from transgenic rice lines with variable Rieske FeS protein content in the cytochrome $b_{6} / f$ complex. Plant.Cell \& Environment. 2016.

30. Aurel M, Schttler, Szilvia Z, et al. Photosynthetic complex stoichiometry dynamics in higher plants: biogenesis, function, and turnover of ATP synthase and the cytochrome $b_{6} / f$ complex. Journal of experimental botany, 2015.

31. Alsharafa, Khalid. Acclimation of normal or low light grown Arabidopsis thaliana to high light. 2012.

32. Kubo S, Masumura T, Saito Y, et al. Cyclic electron flow around PSI functions in the photoinhibited rice leaves. Soil ence \& Plant Nutrition. 2011; 57(1):105-113.

33. Ermakova M, Lopez-Calcagno P E, Raines C A, et al. Overexpression of the Rieske FeS protein of the Cytochrome $b_{6} / f$ complex increases $C 4$ photosynthesis in Setaria viridis. Communications Biology. 2019;2(1):1-12.

34. Yin X, Struik P C. Mathematical review of the energy transduction stoichiometries of C4 leaf photosynthesis under limiting light. Plant Cell \& Environment. 2012;35(7):1299-1312.

35. Anja, Korn, Ghada, et al. Ferredoxin: NADP+ Oxidoreductase Association with Phycocyanin Modulates Its Properties. Journal of Biological Chemistry.2009.

36. Masayoshi S, Shu K, Kaoru K, et al. Gymnosperms have increased capacity for electron leakage to oxygen (Mehler and PTOX reactions) in photosynthesis compared with angiosperms. Plant \& Cell Physiology.2013;(7):1152-1163.

37. Rott Markus, Martins Nádia F, Thiele Wolfram, et al. ATP synthase repression in tobacco restricts photosynthetic electron transport, $\mathrm{CO}_{2}$ assimilation, and plant growth by overacidification of the thylakoid lumen. The Plant cell.2011;23(1).

38. Maroco J P, Rodrigues, M. Lucília, Lopes C, et al. Limitations to leaf photosynthesis in field-grown grapevine under drought-metabolic and modeling approaches. Functional Plant Biology.2002;29:451459.

39. Dias M C, W. Brüggemann. Limitations of photosynthesis in Phaseolus vulgaris under drought stress: gas exchange, chlorophyll fluorescence and Calvin cycle enzymes. Photosynthetica.2010;48(1):96102.

40. Xiaolan R, Dixon R A. The Differences between NAD-ME and NADP-ME Subtypes of C4 Photosynthesis: More than Decarboxylating Enzymes. Frontiers in Plant ence.2016;7. 
41. Mitra S, Srivastava G C. Photosynthesis as Influenced by Assimilate Level in Sunflower (Helianthus annuus L.). Journal of Agronomy and Crop ence. 2010;171(1):20-25.

42. Perdomo J A, Sebastià Capó-Bauçà, Elizabete C S, et al. Rubisco and Rubisco Activase Play an Important Role in the Biochemical Limitations of Photosynthesis in Rice, Wheat, and Maize under High Temperature and Water Deficit. Frontiers in Plant ence.2017;8:490.

43. Rustad T R, Minch K J, Ma S, et al. Mapping and manipulating the Mycobacterium tuberculosis transcriptome using a transcription factor overexpression-derived regulatory network. Genome Biology. 2014;15(502).

44. Yueying, Li, Liangliang, et al. Genome-Wide Identification, Characterization, and Expression Profiling of the Legume BZR Transcription Factor Gene Family. Frontiers in Plant Science.2018.

45. Mushtaq M A, Qi P, Daozong C, et al. Comparative Leaves Transcriptome Analysis Emphasizing on Accumulation of Anthocyanins in Brassica: Molecular Regulation and Potential Interaction with Photosynthesis. Frontiers in Plant ence.2016;7.

\section{Additional Files}

Additional file 1: Figure S1. Species classification.

Additional file 2: Table S1. Total GO enrichment.

Additional file 3: Table S2. KEGG Pathways and Genes Enriched.

Additional file 4: Table S3. J3vsJ0.DEG KEGG pathway enrichment.

Additional file 5: Table S4. J7vsJ0.DEG KEGG pathway enrichment.

Additional file 6: Table S5. J3 vs J0 TF DEG detailed stat.

Additional file 7: Table S6. J7 vs J0 TF DEG detailed stat.

Additional file 8: Table S7. Overview of the test site.

Additional file 9: Table S8. Primers for quantitative RT-PCR.

\section{Figures}



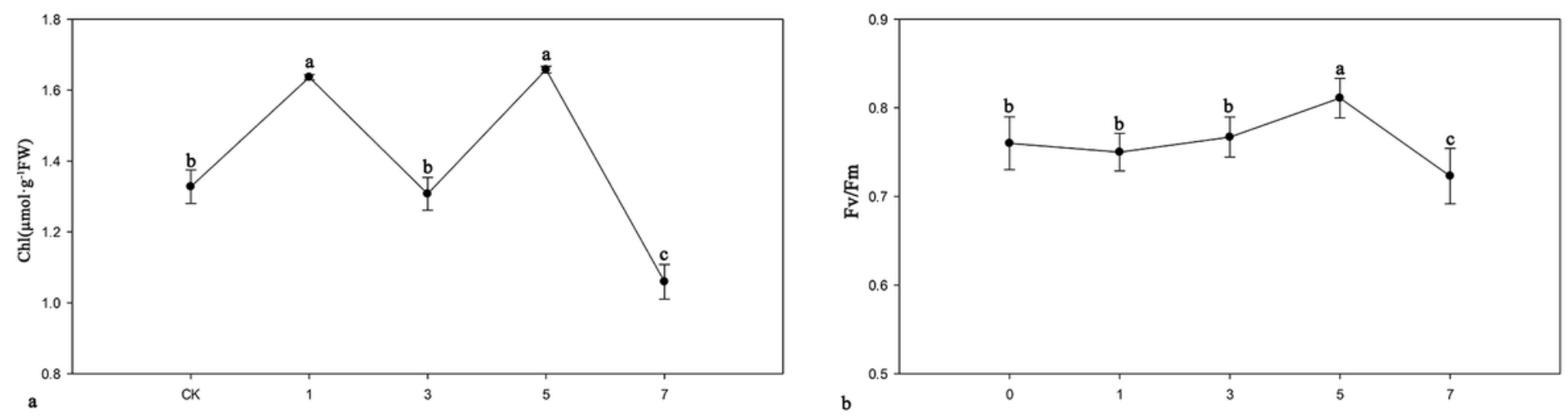

\section{Figure 1}

Changes in chlorophyll content and chlorophyll fluorescence in leaves. a Chlorophyll content and b chlorophyll fluorescence data were collected for the control condition and at 4 different days (days 1, 3, 5, and 7).

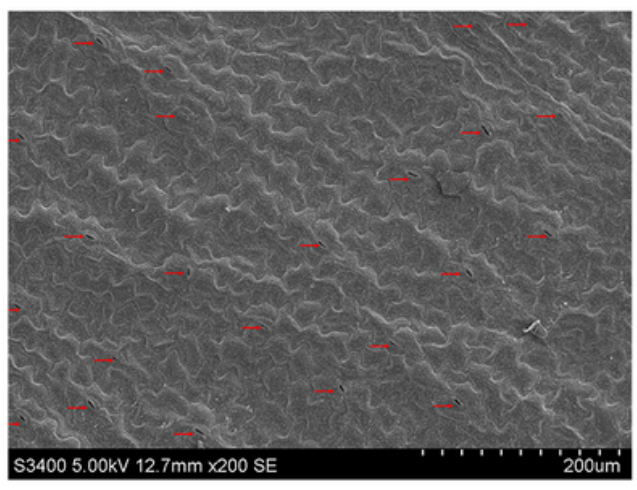

a

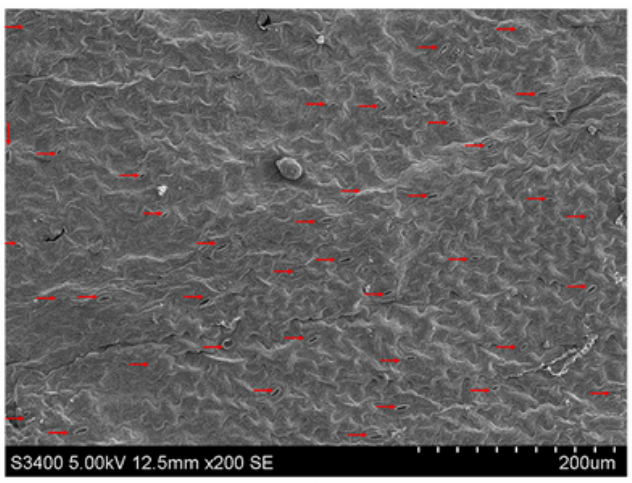

d

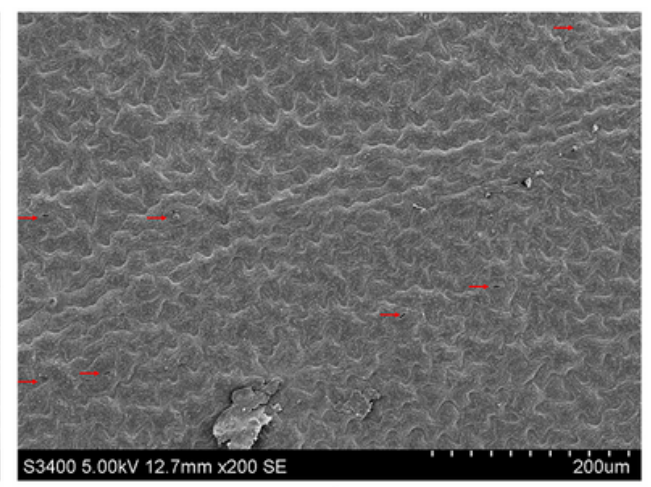

b

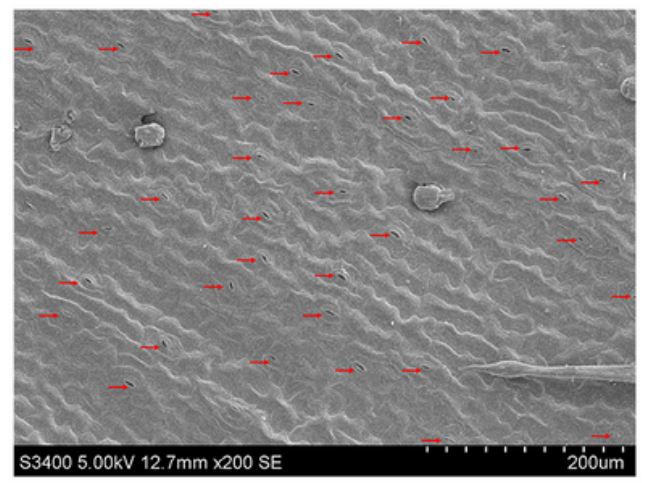

e

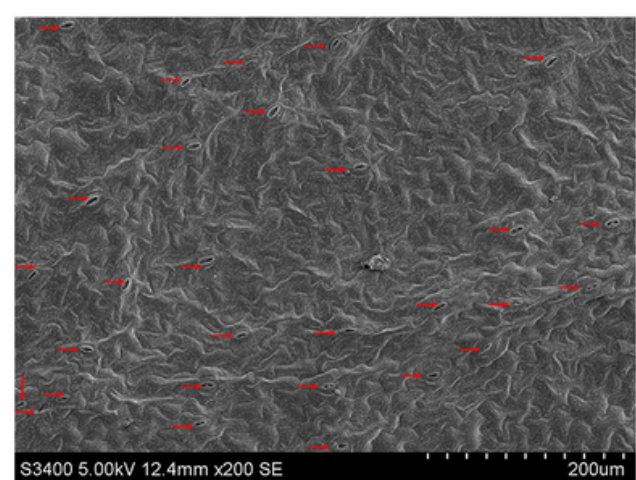

C

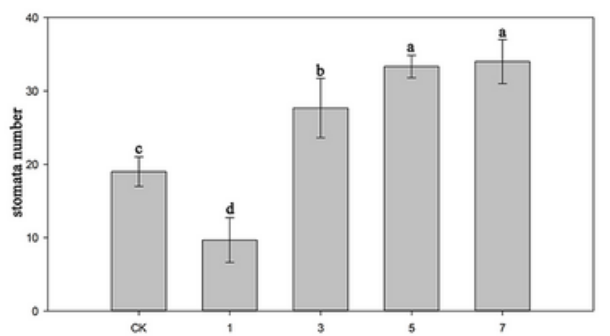

f

\section{Figure 2}

Number of stomata on common vetch leaves under plain conditions and control conditions. Images of the stomata: a control group, b the first day (J1), c the third day (J3), d the fifth day (J5), e the seventh day (J7) and $f$ the number of stomata. Significant differences between the plain condition and control condition are indicated by different lowercase letters $(p<0.05)$ 


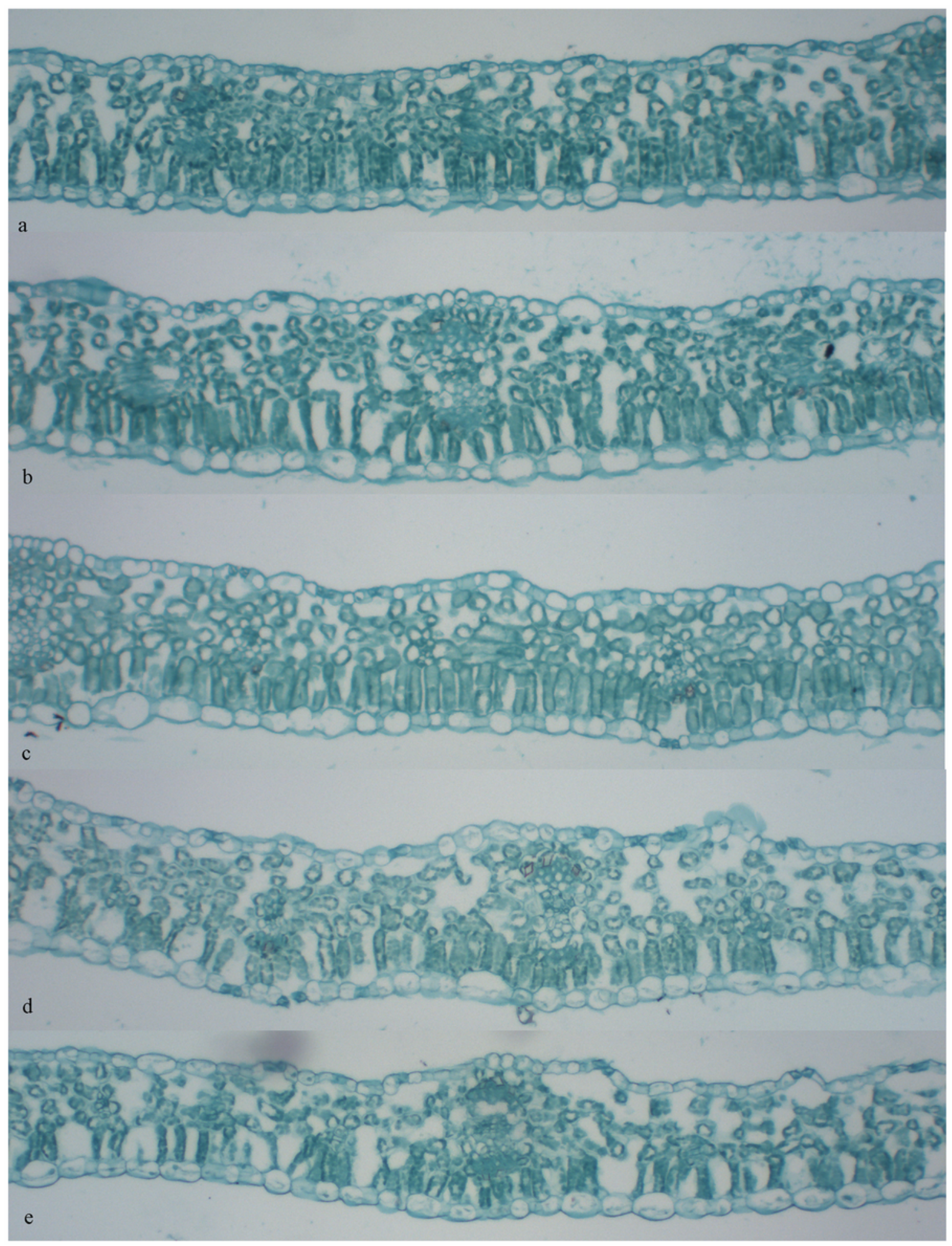

\section{Figure 3}

Anatomical structure of common vetch leaves. a Control group, b the first day (J1), c the third day (J3), d the fifth day (J5), e the seventh day 

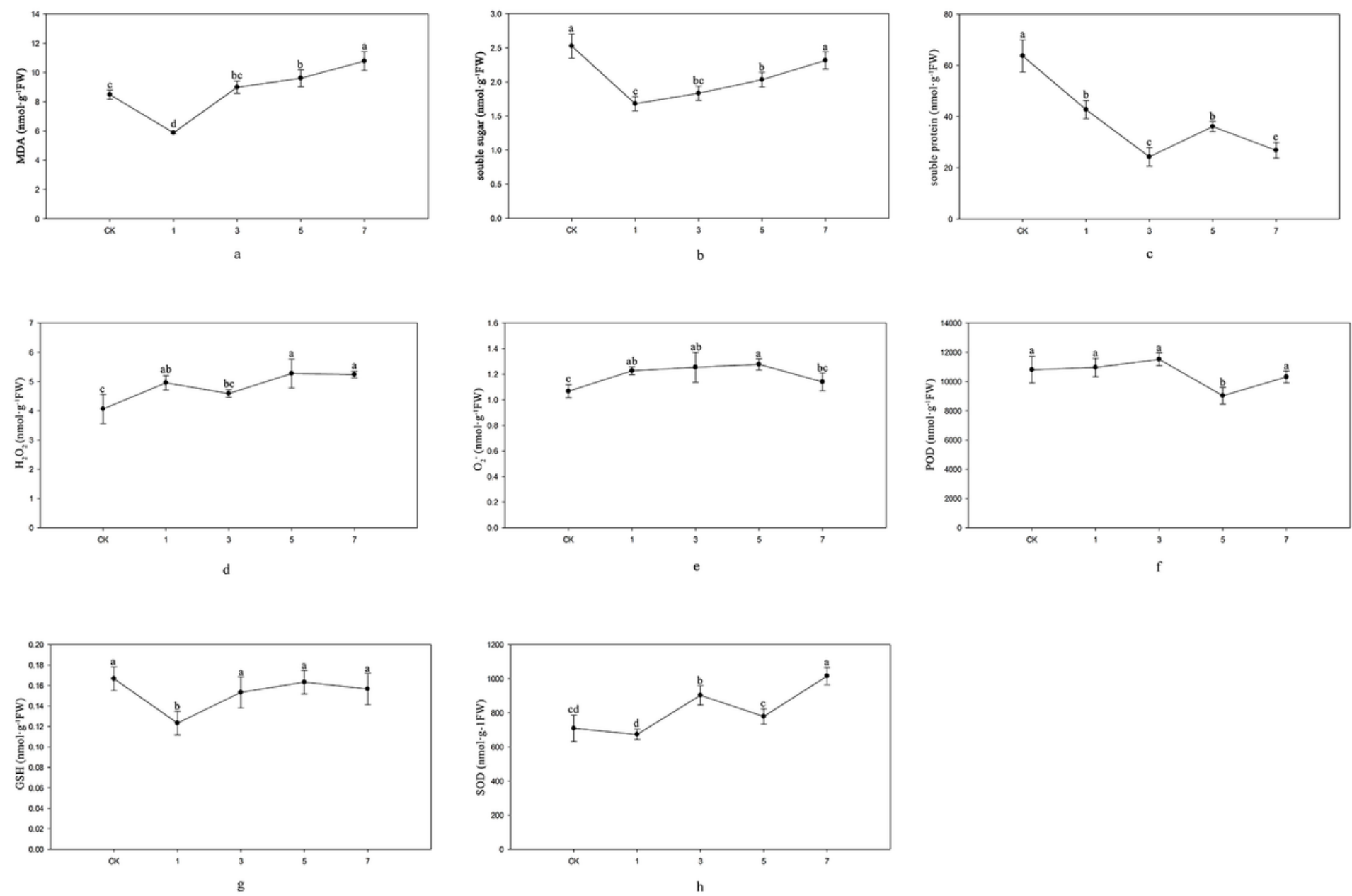

\section{Figure 4}

Physiological changes in common vetch leaves under plain conditions. The contents of MDA a, soluble sugar b, soluble protein c, $\mathrm{H} 2 \mathrm{O} 2 \mathrm{~d}, \mathrm{O} 2-\mathrm{e}, \mathrm{POD} \mathrm{f}, \mathrm{GSH} \mathrm{g}$ and SOD h were determined. Three repetitions were used in the analysis, and significant differences between the plain condition and control condition are indicated by different lowercase letters $(p<0.05)$. 

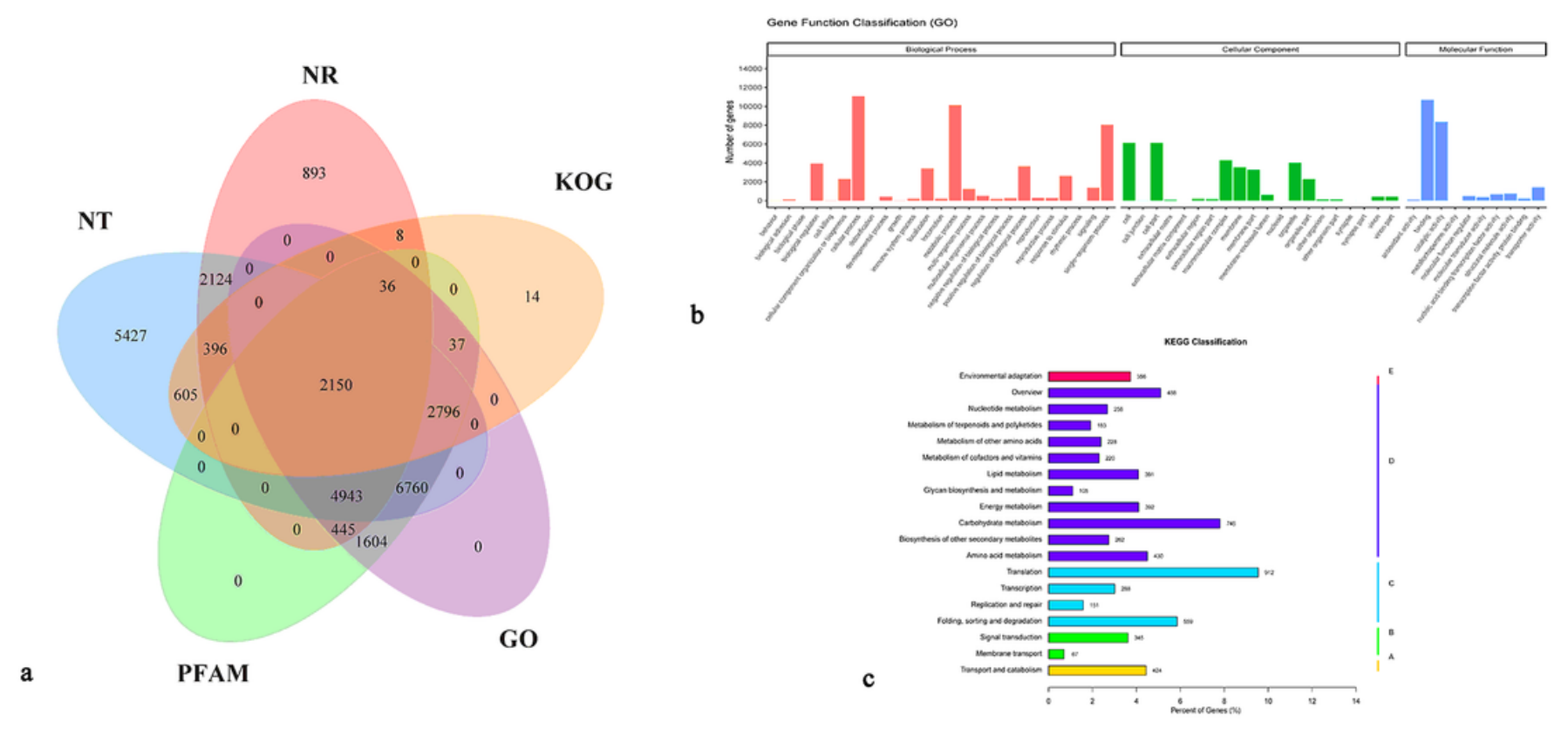

\section{Figure 5}

Annotation and functional classification of unigenes. a Venn diagram of unigenes functionally annotated for leaves. b GO annotation. The $x$-axis is the GO classification of gene function, and the $y$-axis is the number of genes in the category. c KEGG classification. The x-axis is the number of genes in the interpretation, and the $y$-axis is the classification of gene function.

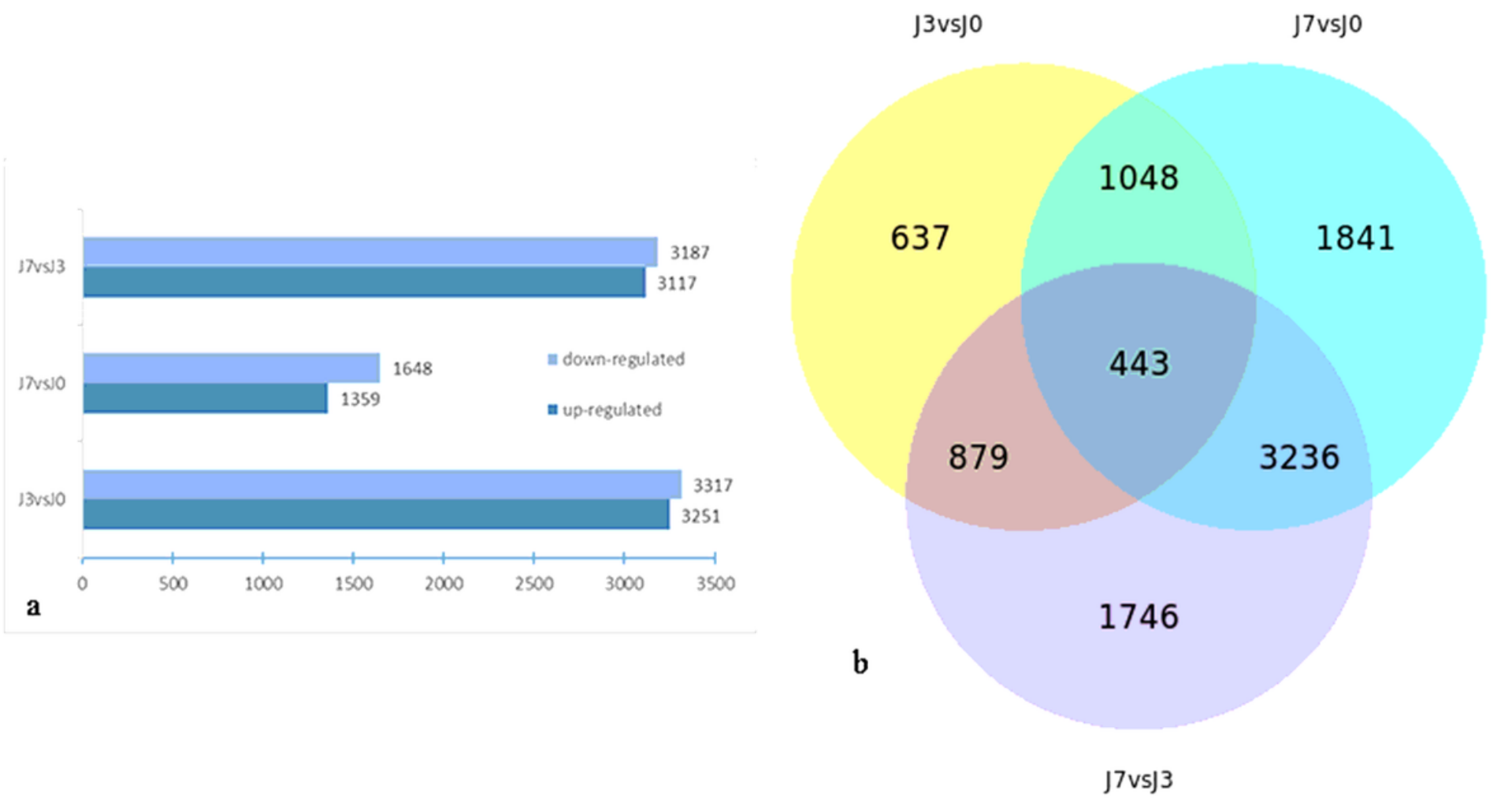


Figure 6

DEGs between the $\mathrm{J} 3$ and $\mathrm{J} 7$ plain samples and the control samples. a Summary of the number of DEGs between the plain and control conditions. $b$ Venn diagram indicating the DEGs identified by J3 vs J0, J7 vs $\mathrm{J} 0$ and $\mathrm{J} 3$ vs $\mathrm{J} 7$ comparisons.
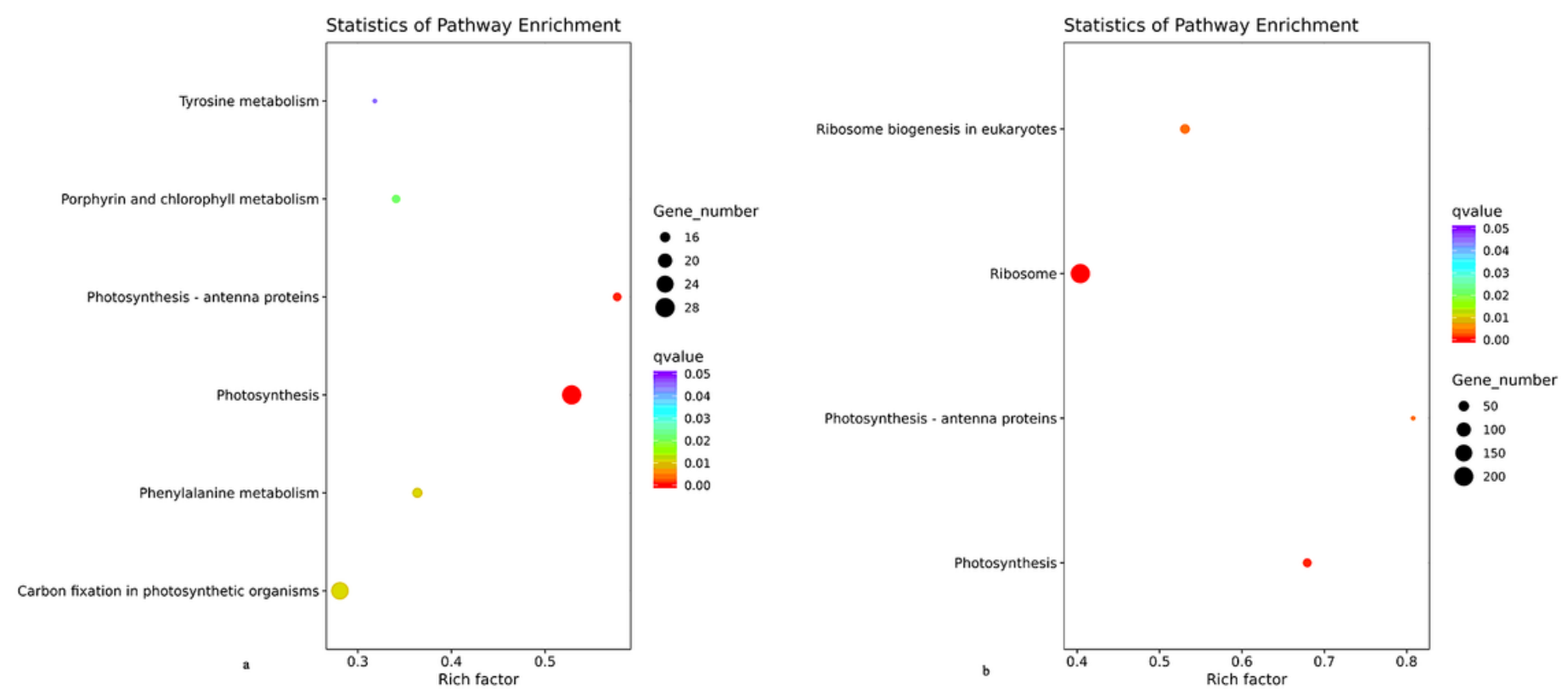

Figure 7

Enriched KEGG pathways of the annotated DEGs. The left Y-axis indicates the KEGG pathway. The X-axis indicates the Rich factor. A high q value is represented by blue. a J3vsJ0, b J7vs J0. 
Cluster analysis of differentially expressed genes

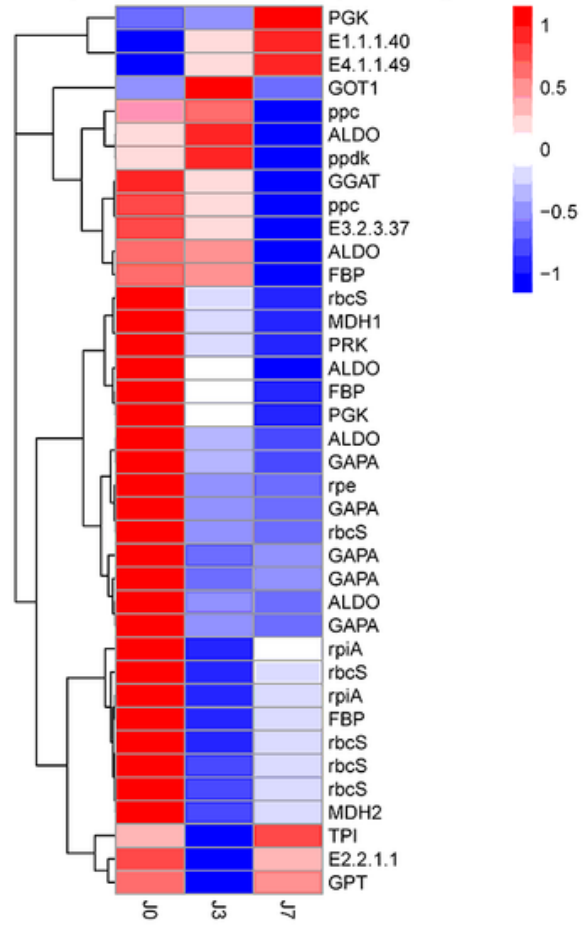

Cluster analysis of differentially expressed genes

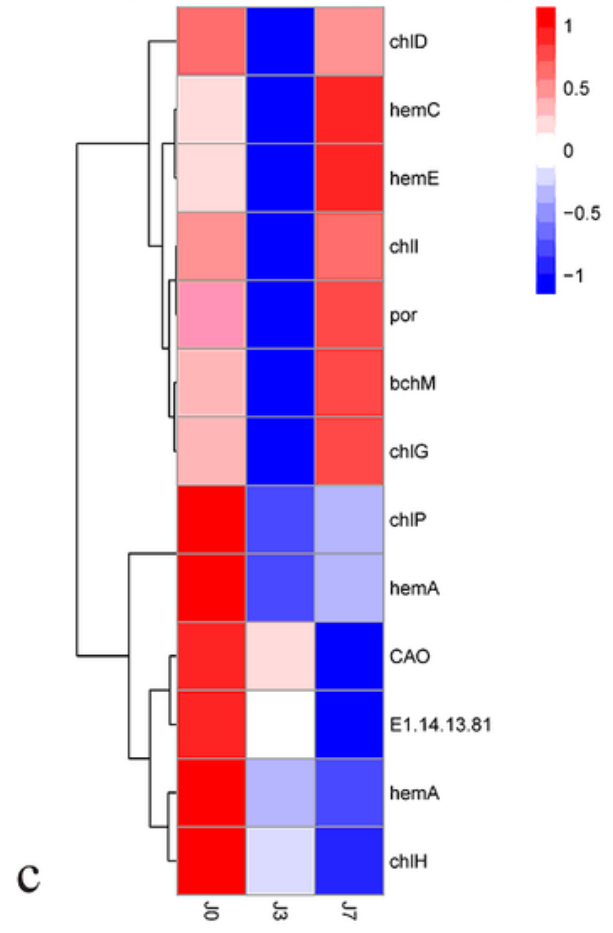

Cluster analysis of differentially expressed genes

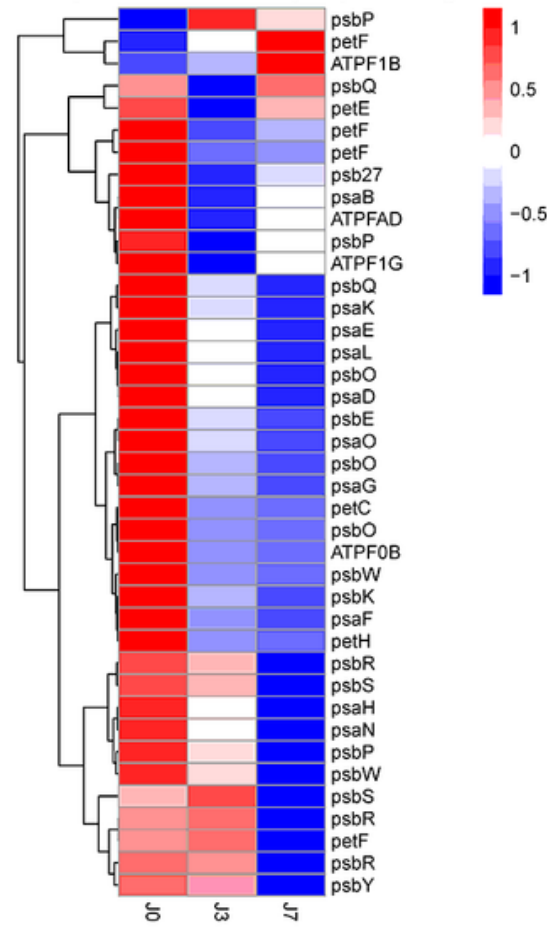

Cluster analysis of differentially expressed genes

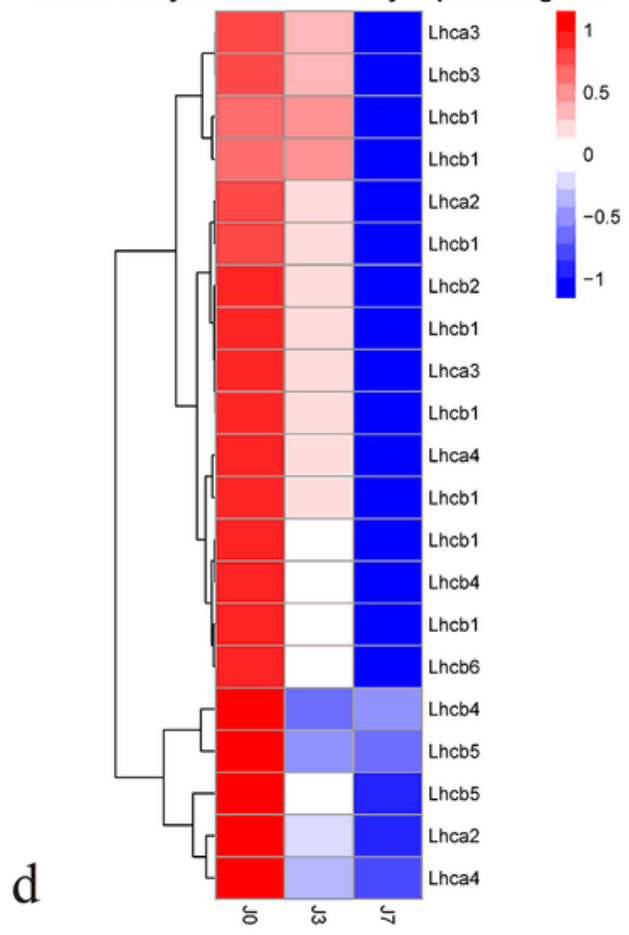

\section{Figure 8}

Heatmap of hub genes in the green module. a Carbon fixation in photosynthetic organisms, $b$ photosynthesis, c porphyrin and chlorophyll metabolism, d photosynthesis - antenna proteins. 


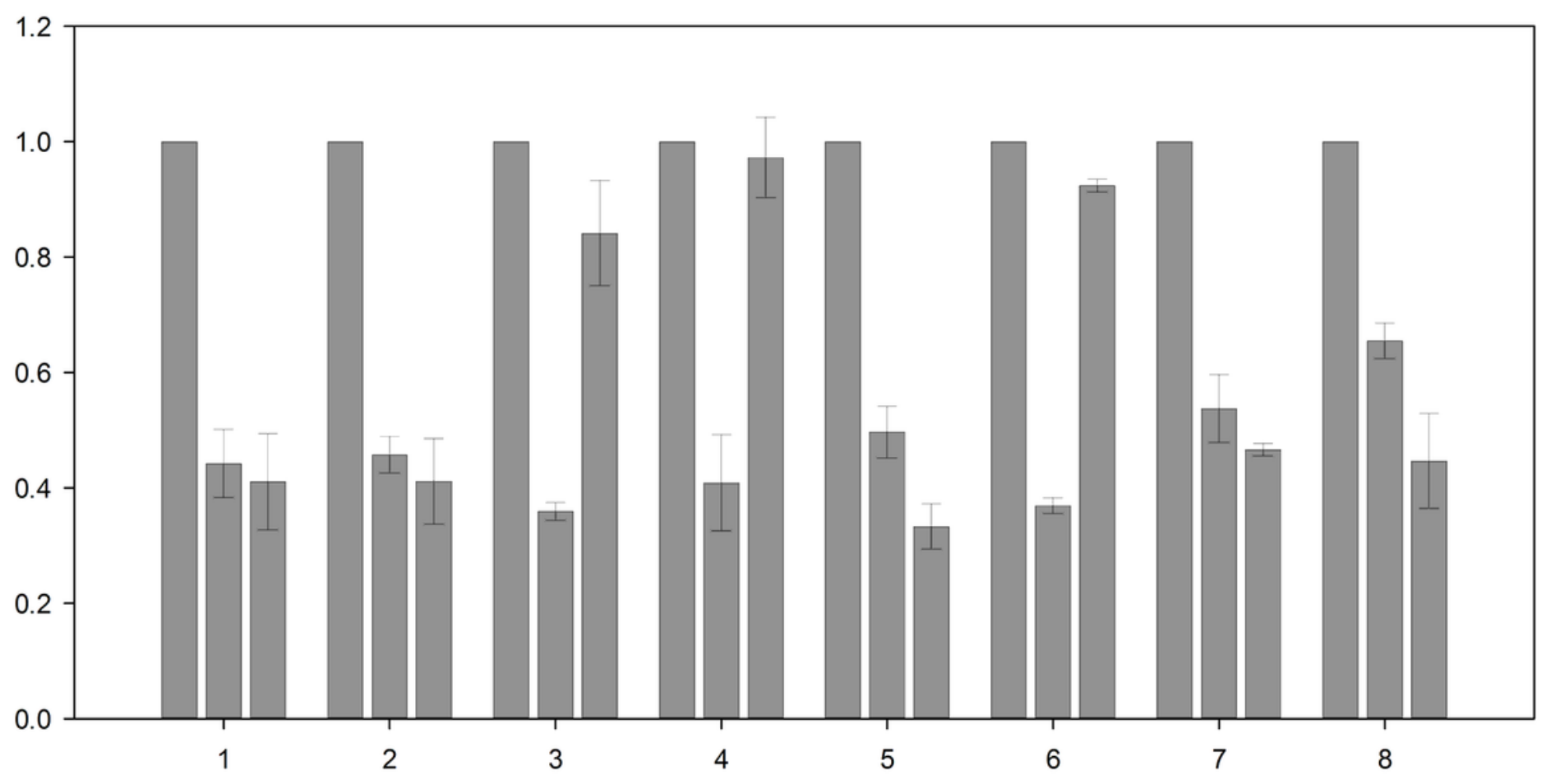

Figure 9

Quantitative RT-PCR confirmation of some downregulated unigenes. Axis $Y$ represents the relative quantification (RQ) between the $\mathrm{J} 3 \mathrm{vs} \mathrm{J} 0$ and $\mathrm{J} 7 \mathrm{vs} \mathrm{J} 0$ treatments, and the error bars indicate the standard deviation of three replicates. 1 (Cluster-7705.13598), 2 (Cluster-7705.13381), 3 (Cluster7705.13418), 4 (Cluster-7705.13113), 5 (Cluster-7705.13726), 6 (Cluster-7705.13438), 7 (Cluster7705.13612), and 8 (Cluster-7705.14151). The three columns are J0, J3 and J7. 


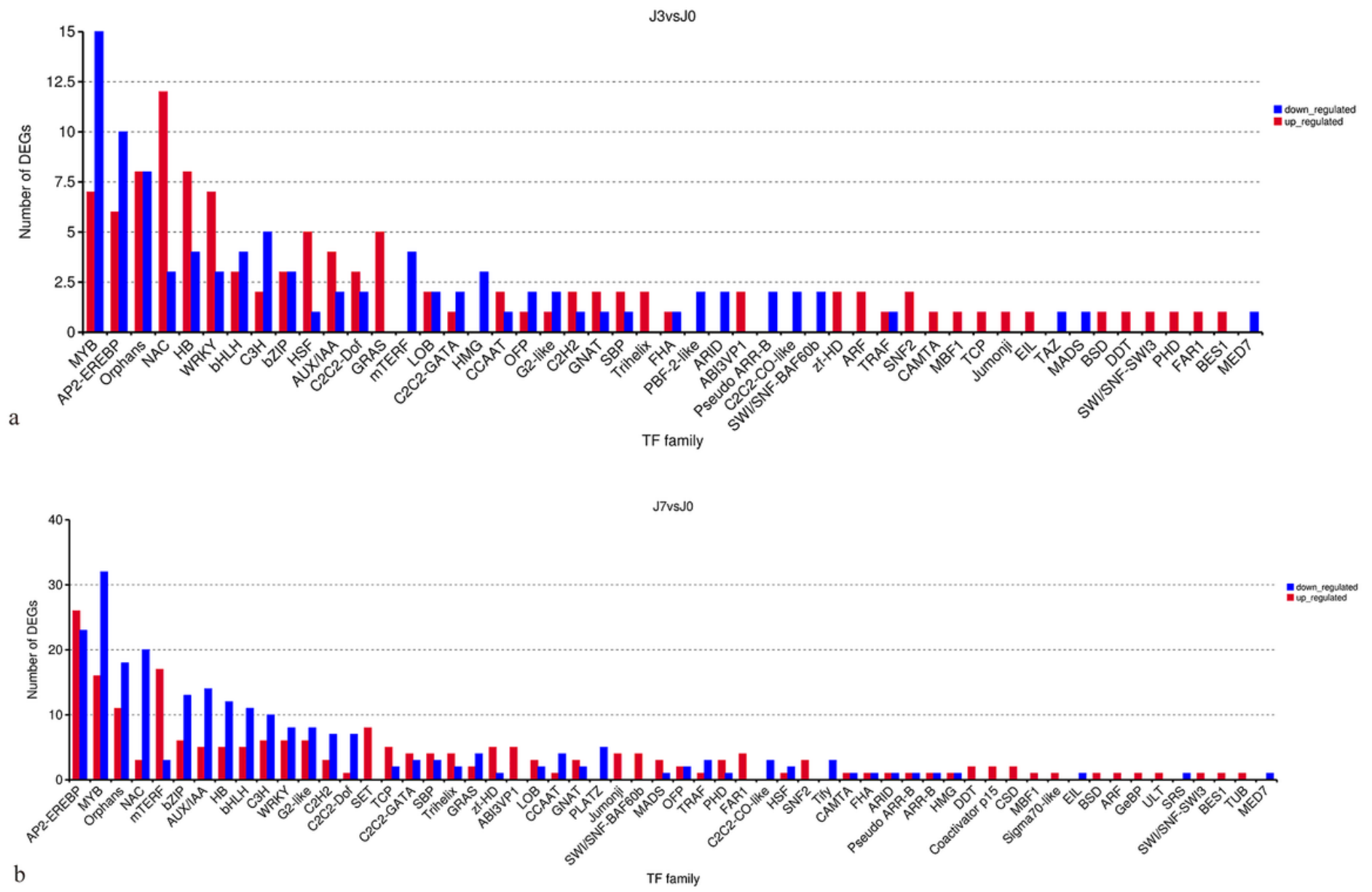

Figure 10

Classification of differentially expressed TFs. a J0 vs J3, b J7 vs J0. Abscissa indicates the TF types; ordinate indicates the number of genes assigned to a specific family 

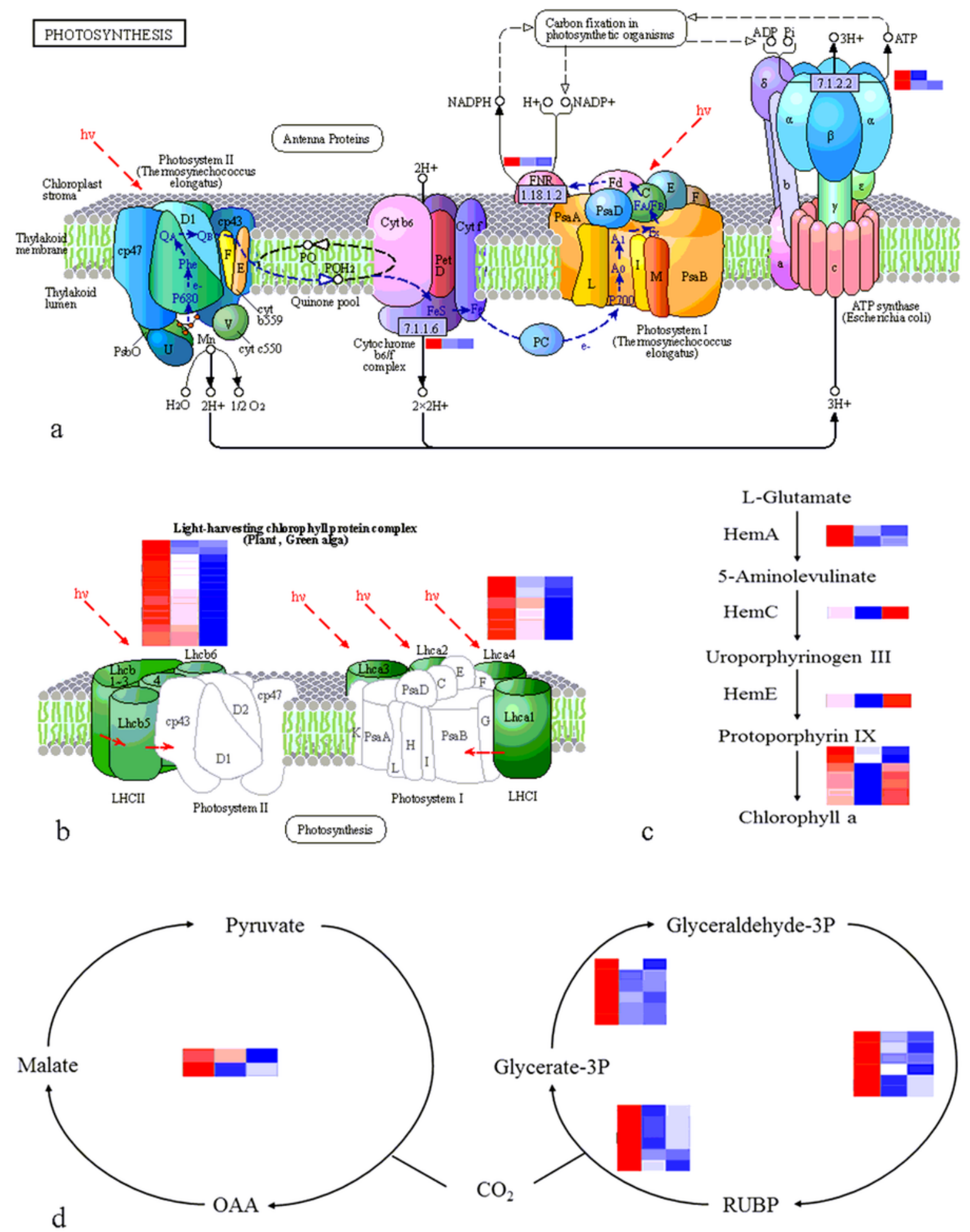

\section{Figure 11}

Responsive progression of gene expression under J0 vs J3 and J7 vs J0. a Photosynthesis, b photosynthesis - antenna proteins, c porphyrin and chlorophyll metabolism, $\mathrm{d}$ carbon fixation in photosynthetic organisms.

\section{Supplementary Files}


This is a list of supplementary files associated with this preprint. Click to download.

- renamed14e6d.xls

- renamed30eab.docx

- renamed30f05.xls

- renamed3cf64.xls

- renamed68ea8.xls

- renamed6a1f0.docx

- renamed6acce.xls

- renamed9f979.docx

- renameda8164.xls 